\title{
EFFECT OF INTRATUMORAL INJECTION OF DOXORUBICIN IN THE TREATMENT OF INDUCED ORAL SQUAMOUS CELL CARCINOMA (EXPERIMENTAL STUDY)
}

\author{
Marwa M. Essawy ${ }^{1} M S c$, Sahar M. El-Sheikh ${ }^{2} P h D$, Hanaa S. Raslan ${ }^{3} P h D$
}

\begin{abstract}
INTRODUCTION: Oral squamous cell carcinoma (OSCC) is the sixth common cancer. Surgery remains the main way of OSCC treatment. It is followed by radiotherapy and chemotherapy either alone or in combination. Conventional chemotherapy is associated with systemic toxicity. Doxorubicin (DOX) is one of the effective chemotherapeutic drugs. Direct delivery of chemotherapeutics may reduce their side effects.

OBJECTIVES: The study aimed to evaluate the anticancer effect of intratumoral injection of DOX in the treatment of chemically induced OSCC.

MATERIALS AND METHODS: Thirty-two Syrian golden male hamsters were used as an animal model for oral carcinogenesis. After cancer induction, the hamsters were divided into 4 groups; saline-intraperitoneal (IP), saline-intratumoral, DOX-IP, and DOX-intratumoral. The clinical evaluation of the proposed treatment included measuring the change in tumor volume before and after treatment, whereas, the histological evaluation included calculating the apoptotic index. The proliferative activity was evaluated immunohistochemically by minichromosome maintenance 3 (MCM3). In all statistical results, a $p<0.05$ was considered significant.

RESULTS: The DOX-IP and DOX-intratumoral groups reported significant decrease in the mean tumor volume. The apoptotic index (AI) was significant only in the DOX-intratumoral group. The MCM3 immunostain showed significant decrease in the values of mean area percent (MA\%) and mean optical density (MOD) in DOX-intratumoral group, whereas the DOX-IP group was insignificant in its MA\% and MOD. CONCLUSION : Local delivery of this chemotherapeutic drug may be suitable alternative to the systemic administration, with superior anticancer effect in the treatment of superficial tumors.

KEY WORDS: Oral squamous cell carcinoma, Chemotherapy, Doxorubicin, Intratumoral, Hamsters, Minichromosome maintenance 3
\end{abstract}

1- Master in Oral Pathology, Oral Pathology Department Faculty of Dentistry, Alexandria University.

2- Professor of Oral Pathology, Oral Pathology Department, Faculty of Dentistry, Alexandria University.

\section{INTRODUCTION}

Cancer is a global health problem of increasing incidence and mortality rates, with 25 million people currently living with cancer. In developed countries, cancer has become the leading cause of death, while in developing countries it is considered the second cause of death after heart diseases $(1,2)$. According to the World Health Organization (WHO) cancer rates are expected to increase at an alarming rate: from 10 million new cases globally in 2000 to 15 million in 2020 (3).

Head and neck cancer is among the 10 most common cancers worldwide (2). It is the sixth most common cancer, with an incidence of over 500,000 new cases each year (4). Approximately $90 \%$ of the head and neck cancers are oral squamous cell carcinoma (OSCC). In the last 30 years, the 5 -year survival rate of patients with OSCC did not improve despite the advances in diagnostic techniques and the improvements in treatment modalities $(5,6)$.

The main treatment for OSCC continues to be surgical intervention followed by radiotherapy and chemotherapy, either alone or in combination, in order to achieve total cure $(7,8)$. Chemotherapy has become increasingly more effective in OSCC treatment. There are several chemotherapeutic drugs for cancer therapy with different mechanisms of action, doses, and routes of administration. Of these agents, doxorubicin (DOX) is widely used in the treatment of a broad spectrum of cancers such as breast, lung, gastric, ovarian, thyroid, non-Hodgkin's and Hodgkin's lymphoma, multiple myeloma, and sarcomas. Doxorubicin is an anthracyline drug first extracted from
Streptomyces peucetius in the 1970's. It is a cytotoxic drug that inhibits the synthesis of nucleic acids within cancer cells. The major side effect of the cumulative dose of DOX is the cardiotoxicity $(9,10)$.

Intratumoral injection of chemotherapeutic drug is another approach to deliver the drug directly to the tumor site, especially the superficial tumors such as OSCC, in an attempt to reduce its systemic toxicity (11). Therefore, the present study evaluated the inratumoral injection of DOX in the treatment of chemically induced OSCC in buccal pouches of Syrian golden hamsters.

\section{MATERIALS AND METHODS}

A blinded prospective comparative study was conducted on Syrian golden hamsters to evaluate the anti-tumor effect of DOX injected intratumorally in induced OSCC.

\section{Sample}

Thirty-two Syrian golden male hamsters (Mesocricetus auratus) five weeks old, weighting $80-129$ g were obtained from Theodor Bilharz Institute, Cairo, Egypt. They were housed in show box cages, at the Experimental Animal Unit in the Medical Research Institute, Alexandria University. Drinking water and conventional feed were provided in accordance to the Medical Research Institute guidelines. The experiment was approved by the Ethical Committee of the Faculty of Dentistry, Alexandria University.

\section{Cancer induction}

An OSCC was induced chemically in the 32 Syrian golden hamsters. The used chemical carcinogen was 7, 12 
dimethylbenz [a] anthracene (DMBA; > 95\%, Sigma Aldrich Chemie GmbH, Germany). It was prepared by dissolving $0.5 \mathrm{~g}$ DMBA in $100 \mathrm{ml}$ paraffin oil. Then, the animals' left buccal pouches were painted with the $0.5 \%$ DMBA, using a brush number 4 . This procedure was repeated 3 times per week for 18 weeks or upon developing tumors $(12,13)$. During carcinogenesis process the hamsters were observed for clinical changes. At the end of cancer induction, four animals were sacrificed for histopathological evaluation of the developed oral tumors.

\section{Grouping}

The remaining 28 hamsters (out of 32 hamsters) were randomly divided into 4 study groups, 7 hamsters per group. The 2 negative control groups were treated with saline; one was intra-peritoneally (saline-IP) and the other was intratumorally (saline-intratumoral). The third group was the positive control that was treated with DOX (EIMC United Pharmaceuticals, Egypt) that was given IP (DOX-IP). The fourth group was the study group that was given the DOX intratumorally (DOX-intratumoral).

Each group received the proposed treatment three times per week for one week. The dose of the injected DOX was $10 \mathrm{mg} / \mathrm{kg}$, whether injected IP or intratumorally (14).

\section{Evaluation of the proposed treatment}

\section{4a. Clinical evaluation}

The tumor volume (TV) was measured before the treatment. It was estimated by measuring tumor length ( $\mathrm{D}$ max: longer dimension) and width (D min: shorter dimension) with a caliper and calculated as: $T V=D \max X(D \text { min })^{2} / 2$ (15).

The same measurements were performed after the treatment over a period of 4 weeks. The percentage of tumor volume change was calculated as: TV Change $\%=[(T V$ after treatment - TV before treatment) / TV before treatment] X 100 (16).

4b. Microscopic evaluation

\section{i. Apoptosis detection}

The hamsters were sacrificed by an overdose of ketamine hydrochloride ( $>30 \mathrm{mg} / \mathrm{kg}$ ), given IP. The tumor masses from the sacrificed animals were processed for routine hematoxylin and eosin (H\&E) stain to detect the apoptotic cells and counting the apoptotic index (AI).

The cells were considered apoptotic according to the standard criteria for apoptosis; dense condensed nodular hyperchromatic nuclei, fragmentation of chromatin, deep eosinophilia of the cytoplasm, cell shrinkage, and absence of lymphocyte infiltration and inflammation. Single and roundish nuclear residues existing in extra or intratumoral cells with intensive staining were identified as apoptotic bodies. Obvious necrotic areas were excluded from the analysis $(17,18)$.

Apoptotic cells were counted from at least 10 randomly chosen high-power fields. The AI was calculated from the ratio of the number of apoptotic cells to the total number of tumor cells counted per section. At least 1000 cells were counted under magnification of X400 (19).

\section{ii. Immunohistochemical analysis}

Specimens of tumor tissue from the study groups were stained using proliferative marker; minichromosome maintenance 3 (MCM3; Gene Tex, USA), to compare the proliferation rates among study and control groups.

Immunostaining was performed on $4 \mu \mathrm{m}$ paraffin sections. Sections were de-paraffinized with xylene and rehydrated in graded ethyl alcohol. Before staining procedure, samples were immersed in citrate buffer solution $(\mathrm{pH}=6)$. Endogenous peroxidase activity was blocked with $3 \%$ hydrogen peroxide for 3 minutes. Heat induced epitope retrieval was done by boiling the sections in citrate buffer solution for 10 minutes. Then the sections were cooled for 20 minutes. To evaluate the MCM3 antigen expression, mouse monoclonal antibodies were used (clone Rb pAb GTX111102; 1:100; Gene Tex). Sections were incubated with primary antibody and then were washed in phosphate-buffered saline (PBS). Finally, secondary antibody associated with Envision System was applied. Sections were washed in PBS again. The 3,3 diaminobenzidine was applied as a chromogen for antibody detection. Sections were counterstained with Mayer's hematoxylin. Negative control was a section of the same sample where the primary antibody was replaced by PBS. The epithelial layer of normal oral mucosa that has previously shown immunostaining of MCM3 was considered as positive controls (20).

The sections were examined in randomly selected microscopic fields at a magnification of X400. The intensity of immunostaining of MCM3 was calculated in terms of mean area percent (MA\%) and mean optical density (MOD) by the computer image analyzer.

\section{STATISTICAL ANALYSIS}

The data were collected, tabulated and statistically analyzed using the SPSS system (release 11.0 software). All results were expressed as mean \pm standard deviation (SD). Tumor volume changes before and after treatment was analyzed statistically using paired $t$-test.

One-way ANOVA was used to analyze the AI of the $\mathrm{H} \& \mathrm{E}$ results between study groups. Also it was used to analyze the MA\% and MOD of immunohistochemical results. In all statistical results, a $p<0.05$ was considered significant.

\section{RESULTS}

\section{Cancer induction}

\section{1a. Clinical changes during carcinogenesis}

The OSCC was chemically induced in the left pouches of hamsters, following a standardized carcinogenesis protocol, using $0.5 \%$ DMBA in liquid paraffin. Before cancer induction, the normal hamster buccal pouch (HBP) was pale pink in color. During carcinogenesis period, the buccal mucosae revealed sequential clinical changes. They ranged from leukoplakic to erythroplakic non-scarpable patches that appeared after $10-18$ weeks of cancer induction.

The well-developed oral tumor masses started to appear after 20 - 24 weeks of carcinogenesis. They ranged from endophytic ulcers with necrotic floor to exophytic masses with granular surfaces, (Figure 1). Some of the hamsters developed multiple intraoral tumor masses.

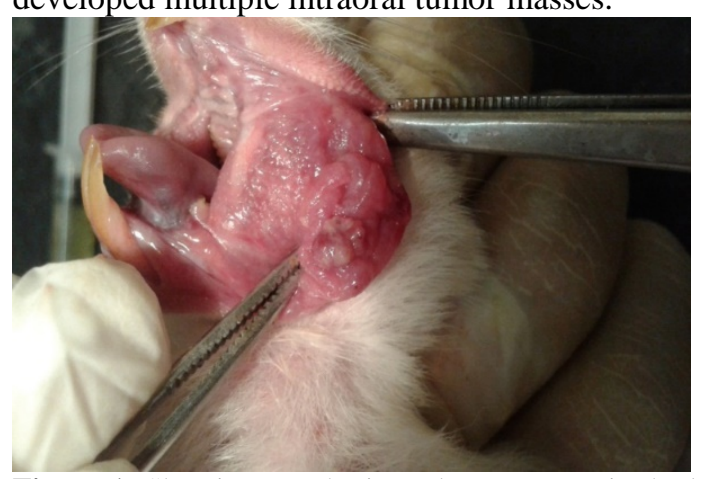

Figure 1: Showing exophytic oral tumor mass in the left hamster buccal pouch after cancer induction. 
By the end of carcinogenesis process, 5 animals died during the experiment period. Out of the remaining 27 hamsters, 3 hamsters were sacrificed for histopathological evaluation of the tumor masses.

\section{1b. Histopathological evaluation}

The normal HBP was extracted from the unpainted right side. The wall of the buccal pouch consisted of 3 distinct layers; the thin corrugated lining keratinized stratified squamous epithelium, the loose fibrous subepithelial connective tissue, and the tunica muscularis. Neither inflammatory cells nor lymphatic vessels were seen.

By the end of carcinogenesis period, the extracted oral tumors revealed SCC with different grades of differentiation; well, moderately, and poorly differentiated SCC. The well differentiated SCC was consisting of keratin pearls and epithelial pearls. In moderately differentiated SCC, the malignant epithelial cells were arranged in cell nests and few epithelial pearls. The poorly differentiated SCC revealed cell nests intermingled with sheets of anaplastic cells (Figure 2).

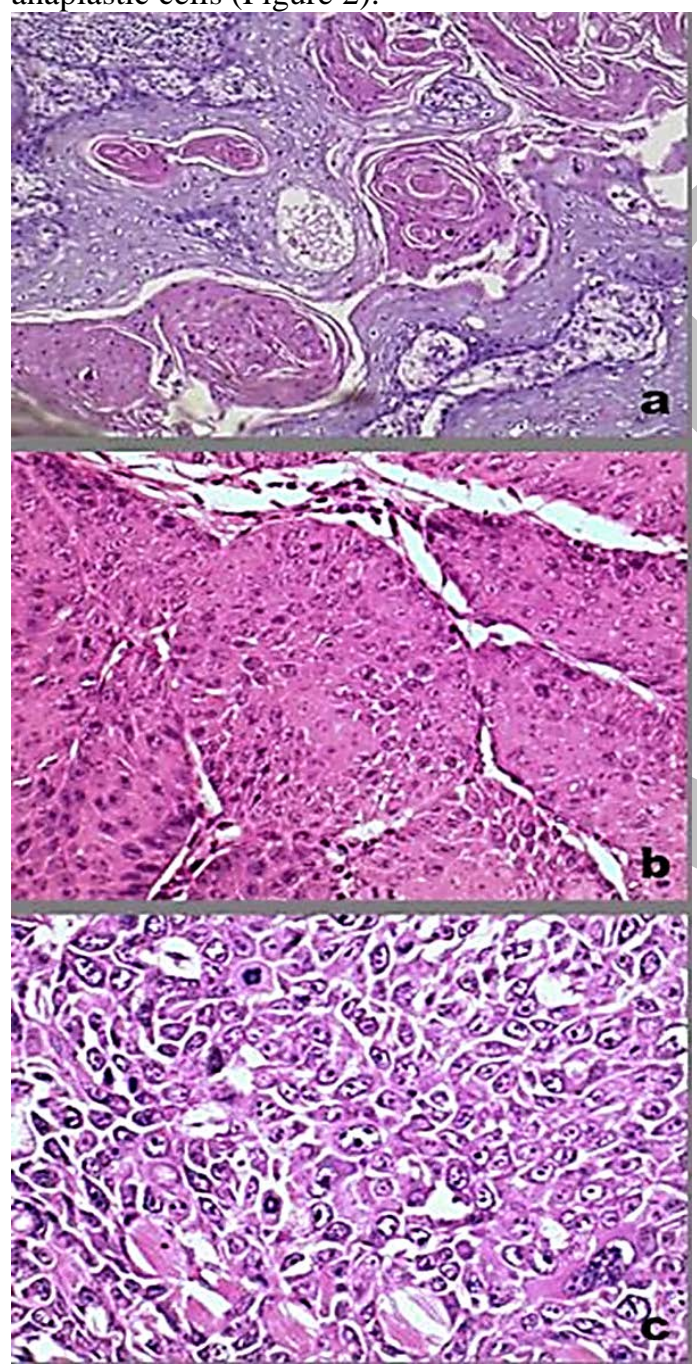

Figure 2: Showing different histopathological grades of oral squamous cell carcinoma (SCC) developed after cancer induction. a: Well differentiated SCC. b: Moderately differentiated SCC. c: Poorly differentiated SCC. (a-c: Hematoxylin and eosin, X400).

\section{Grouping and treatment plan}

The remaining 24 hamsters developed tumor masses with incident rate of $100 \%$ and tumor multiplicity of $1.27 \pm 0.62$ tumors per hamster. They were divided randomly into 4 study groups ( $n=6$ each group), according to the type of the proposed treatment and the route of its administration;
saline-IP, saline-intratumoral, DOX-IP, and DOXintratumoral.

\section{Clinical evaluation of the proposed treatment}

The tumor volumes in the study groups recorded changes at the $4^{\text {th }}$ week after treatment. In the 2 negative control saline groups, some tumors continued to increase in their volume by a percent change of $6.2 \pm 2.5$ in the IP group and $7.8 \pm 8.5$ in the intratumoral group. On the other hand, the tumors in the 2 DOX groups showed decrease in their mean volumes after treatment. The DOX-IP treated group showed decrease in their mean tumor volumes by $-8.1 \pm 1.6$, whereas the highest percent of mean volume decrease was recorded in the DOXintratumoral injected group that was - $20.2 \pm 13.3$, (Figure 3).

At the $4^{\text {th }}$ week after treatment, the mean tumor volume decreased significantly compared to that recorded before treatment in the groups treated with DOX, whether IP or intratumorally $(p<0.05)$.

The mean tumor volume before and after treatment with the percent of tumor volume change are summarized in Table 1.

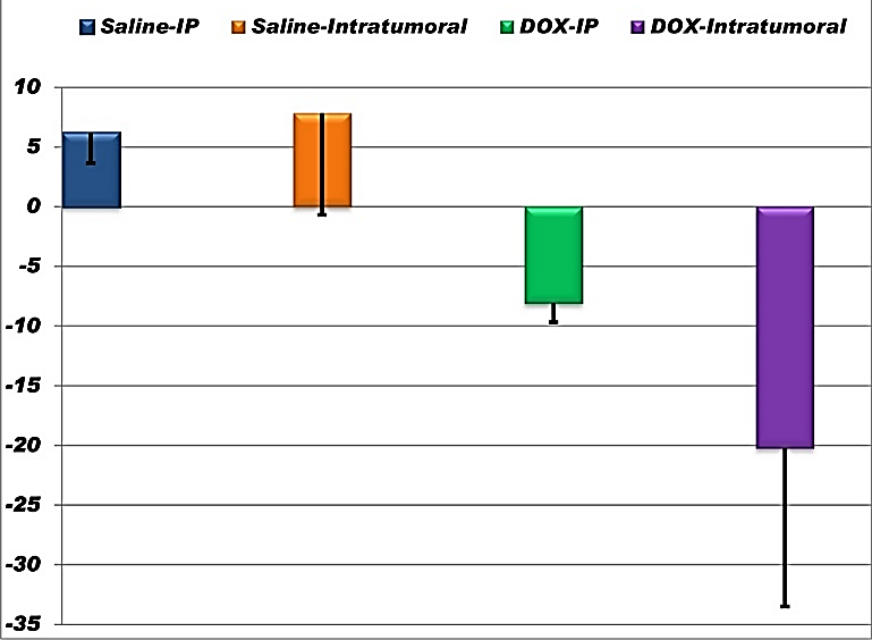

Figure 3: Showing percentages of tumor volume changes in all groups after 4 weeks from treatment (mean of decrease or increase \pm SD).

Table 1: Tumor volume in $\mathrm{mm}^{3}$ (mean \pm SD) before and after treatment by 4 weeks, percentage of volume change (mean \pm SD).

\begin{tabular}{|c|c|c|c|}
\hline Groups & $\begin{array}{l}\text { Mean } \\
\text { tumor } \\
\text { volume } \\
\text { before } \\
\text { treatment }\end{array}$ & $\begin{array}{l}\text { Mean } \\
\text { tumor } \\
\text { volume } \\
\text { after } \\
\text { treatment }\end{array}$ & $\begin{array}{l}\text { Percentage } \\
\text { of volume } \\
\text { change }\end{array}$ \\
\hline Saline-IP & $\begin{array}{l}141.0 \quad( \pm \\
90.1)\end{array}$ & $\begin{array}{l}148.2 \quad( \pm \\
91.2)\end{array}$ & $6.2( \pm 2.5)$ \\
\hline $\begin{array}{l}\text { Saline- } \\
\text { Intratumoral }\end{array}$ & $\begin{array}{l}324.3 \quad( \pm \\
333.5)\end{array}$ & $\begin{array}{l}335.5 \\
335.7)\end{array}$ & $7.8( \pm 8.5)$ \\
\hline DOX-IP & $\begin{array}{l}134.0 \\
52.4)\end{array}$ & $\begin{array}{l}123.7 \\
50.6) \dagger\end{array}$ & $-8.1( \pm 1.6)$ \\
\hline $\begin{array}{l}\text { DOX- } \\
\text { Intratumoral }\end{array}$ & $\begin{array}{l}595.8 \\
572.5)\end{array}$ & $\begin{array}{l}521.1 \\
545.4) \dagger\end{array}$ & $\begin{array}{l}-20.2 \quad( \pm \\
13.3)\end{array}$ \\
\hline
\end{tabular}

$\dagger$ Statistically significant $(P<0.05)$ in comparison between mean tumor volume before and after treatment in the same subgroup 


\section{Microscopic evaluation of the proposed treatment 4a. Apoptosis analysis}

Under the light microscope, the different stages of apoptosis were revealed by the treated malignant epithelial cells. In early stages of apoptosis, some epithelial cells especially those at the basement membrane, showed deep eosinophilic cytoplasm surrounding nodular hyperchromatic nuclei. These cells were detached from the adjacent basal cells. Other malignant apoptotic epithelial cells were pyknotic with cytoplasmic clearance. Some nuclei showed condensed chromatin, while others revealed chromatin margination.

In late stages of apoptosis, the apoptotic bodies, which are the residues of apoptotic cells, were seen as single rounded nuclear residues with intensive eosinophilic staining. Absence of inflammatory cells was a character in all fields where apoptosis was examined, (Figure 4).

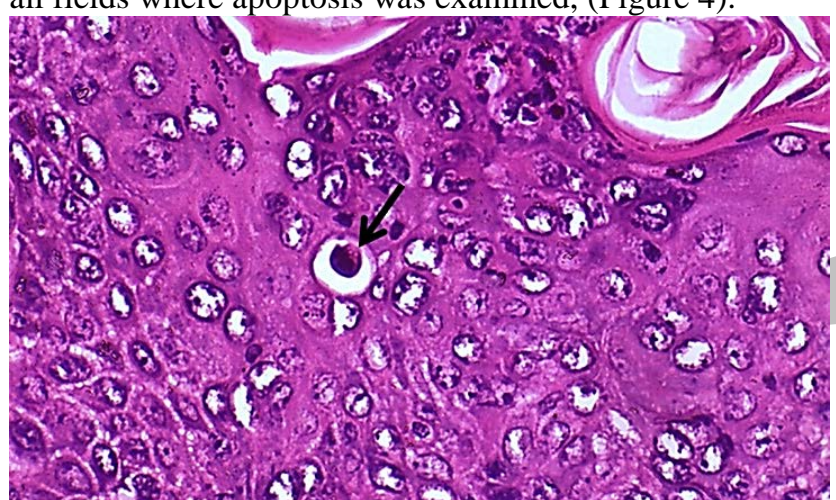

Figure 4: Showing late stage of apoptosis in treated malignant epithelial cells: Apoptotic eosinophilic body (black arrow). Hematoxylin and eosin, X400.

The quantitative AI analysis in the 2 saline control groups revealed mean values of $2.5 \pm 0.45$ in IP group and $2.3 \pm 0.57$ in intratumoral group. The DOX group that was treated IP recorded AI of $4.5 \pm 1.05$ that was of insignificant increase ( $p>$ $0.05)$. The increase of AI was of significant value of $9.07 \pm 0.9$ in the DOX-intratumoral treated group, $(p<0.05)$, (Figure 5).

$\square$ Saline-IP $\square$ Saline-Intratumoral $\square$ DOX-IP $\square$ DOX-Intratumoral

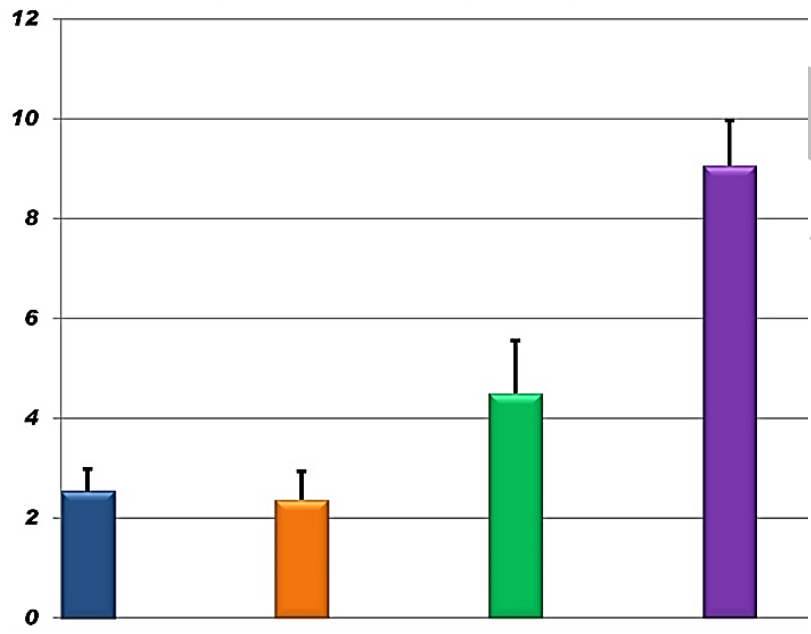

Figure 5: Showing apoptotic indices among the study groups after treatment (mean $\pm \mathrm{SD})$.

\section{4b. Immunohistochemical evaluation}

Generally, the malignant epithelial cells showed nuclear immunoreaction to MCM3. However, the groups showed immunreactivity variations in both mean area percent (MA\%) and mean optical density (MOD), (Figure 6). The 2 control saline groups revealed the most intense positive nuclear stain, with the highest MA\% of $93.2 \pm 2.7$ in IP group, and the highest MOD of $85.6 \pm 65.3$ in intratumoral group.
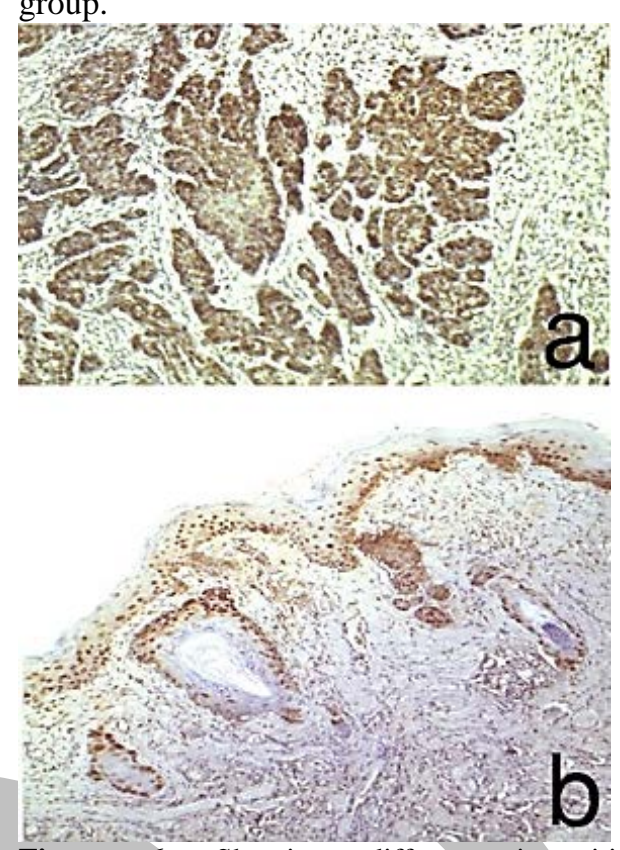

Figure 6: Showing different intensities of MCM3 immunohistochemical stain in treated groups. a: Intense stain in saline-IP group. b: Moderate stain in DOX-intratumoral group. (a and b: MCM3, X100).

In the DOX-IP treated group the immunoreactivity was still intense, despite the decrease in the value of MA\% to $81.4 \pm 3.2$ and MOD to $77.0 \pm 69$.3. However, this decrease in the values was insignificant $(p>0.05)$. The DOXintratumoral treated group showed significant decrease in the MCM3 immunoreaction of $59.7 \pm 6.1 \mathrm{MA} \%$ and 52.3 \pm 43.7 MOD, $(p<0.05)$.

The MA\% and the MOD of the MCM3 imuunostain for the study groups after treatment are summarized in Table 2.

Table 2: Mean area percent $( \pm$ SD) and mean optical density ( \pm $\mathrm{SD})$ of the immunoreaction of MCM3.

\begin{tabular}{|l|l|l|}
\hline Groups & $\begin{array}{l}\text { Mean area } \\
\text { percent }( \pm \text { SD) }\end{array}$ & $\begin{array}{l}\text { Mean optical } \\
\text { density ( } \pm \text { SD) }\end{array}$ \\
\hline Saline-IP & $93.2( \pm 2.7)$ & $83.9( \pm 58.1)$ \\
\hline $\begin{array}{l}\text { Saline- } \\
\text { Intratumoral }\end{array}$ & $87.2( \pm 3.6)$ & $85.6( \pm 65.3)$ \\
\hline DOX-IP & $81.4( \pm 3.2)$ & $77.0( \pm 69.3)$ \\
\hline $\begin{array}{l}\text { DOX- } \\
\text { Intratumoral }\end{array}$ & $59.7( \pm 6.1) \dagger$ & $52.3( \pm 43.7) \dagger$ \\
\hline \hline
\end{tabular}

\section{DISCUSSION}

Oral SCC remains one of the most difficult malignancies to control because of its high ability for local invasion and metastasis. Despite the advances in therapeutic strategies, the survival rates have not changed during the last 20 years, with high mortality rates; about $50-70 \%$ of patients die within 5 years $(21,22)$. Chemotherapy becomes of increasing importance in the treatment of OSCC. It is often 
reserved for metastatic and recurrent tumors. However, the morbidity associated with the chemotherapy impairs the quality of life and may interrupt the cancer treatment $(23,24)$.

Conventionally, chemotherapeutics are given systemically, where they are delivered to the site of tumor passively. They depend on the enhanced permeability and retention (EPR) effect, a mechanism identified by Matsumura and Madea (25). In this EPR mechanism, the "leaky" defective vascular architecture and the poor lymphatic drainage with the slow venous return are responsible for the passive drug delivery, as documented by several studies of Madea et al. (26-28).

Passive targeting approaches however, suffer from several limitations. Targeting cancer cells using the EPR effect is not feasible in all tumors, because the degree of tumor vascularization can vary with the tumor type. Furthermore, lack of controlled targeting can lead to drug expulsion that induces cancer cells to develop resistance toward the drugs, which inevitably reduces their therapeutic effects. Therefore, localized delivery of the drug to the tumor site may overcome these limitations, especially for the local superficial tumors, such as OSCC (29).

The intratumoral injection approach reported success in the treatment of different types of tumors in the field of targeted immunotherapy $(30,31)$. In one of these in vivo studies, Dubrot et al. combined the effect of the immunotherapeutic agent (anti-CD 137) injected IP and chemotherapeutic agent (Interferon) injected intratumorally. They reported synergistic effect in the treatment of subcutaneous tumor derived from the MC38 colon carcinoma, which is resistant to each treatment if given separately (32).

The promising results of these studies open the gate in front of the treatment of cancers with chemotherapeutic drugs that are intratumorally administrated. Panels of chemotherapeutics are available for cancer therapy, each with its specific action. Doxorubicin is one of the cytotoxic antibiotics that are widely used as anticancer drugs. It is routinely used in the treatment of several cancers (9).

In OSCC, DOX exhibits high tumor-specific cytotoxic activity. It is found to induce apoptosis through the activation of caspase-3, -8 and -9 in vitro in OSCC cell line (HSC-2). However, the major limitation for the in vivo use of DOX is the reported cardiotoxicity (33). Therefore, the current study evaluated the intratumoral delivery of DOX in the treatment of chemically induced OSCC in hamster buccal pouches.

In the present study, the clinical evaluation of the proposed treatment reported the highest significant decrease in the mean tumor volume in the group treated intratumorally with DOX, with percent change of $-20.2 \pm 13.3$ in comparison to the other treated groups. This confirms the effective delivery of the DOX to the cancer cells. The DOX-IP treated group reported also significant decrease in the mean tumor volume. However, the percent change was of low value of $-8.1 \pm 1.6$. This could be accomplished to the delivery of DOX through the EPR effect, but with poor drug diffusion to the cancer cells. Meanwhile, the tumors in the 2 control saline groups continued to increase in their volumes.

The anti-cancer effect of the DOX delivered intratumorally at the cellular level was documented by the highest significant AI of $9.07 \pm 0.9$, whereas the $\mathrm{AI}$ in the DOX-IP treated group was insignificant. This confirmed the superiority of intratumoral route of administration to synergize the apoptotic effect of DOX. These results were further confirmed by the decrease in the proliferative activity of the tumors treated by DOX intratumorally. This was done by immunohistochemical analysis, using MCM3 proliferative immune marker, where both the MA\% and the MOD recorded significant decrease. On the other hand, the DOX-IP treated group recorded insignificant decrease in both MA\% and MOD.

In a similar approach of intratumoral injection of chemotherapeutic but with different agent, Zhang et al. treated mouse model of hepatocellular carcinoma. They injected the tumor with octreotide (OCT), tumor growth inhibitor, loaded on poloxamer 407 (P407), temperature-sensitive gel. They reported similar results of that intratumoral injection of OCT-P407 revealed significant reduction in tumor sizes with higher rate of tumor inhibition. Also, the ability of intratumoral injection of OCT-P407 to induce apoptosis was reported by the significant detection of caspase-3, immunohistochemically and by real-time polymerase chain reaction (34).

In another study, Shikanov et al. reported the effectiveness of the intratumoral injection of cisplatin, a chemotherapeutic agent, in the treatment of heterotopic mouse bladder tumor model in mice. They injected the tumors with cisplatin incorporated into a fatty acid-based polymer carrier. This mode of treatment resulted in a successful tumor growth inhibition, with significant decrease in the plasma level of the platinum concentration. The intratumoral delivery system reported increasing in the maximum tolerated dose of cisplatin 5 times compared to systemic delivery, thus potentially improving antitumor efficacy of cisplatin in solid tumor model (35).

On contrary the intravenously delivery of DOX reported significant results in the treatment of OSCC as reported by Chen et al. However, this may be resulted from loading the DOX with paclitaxel, another chemotherapeutic drug, on nanoparticles that were actively targeting multidrug resistant OSCC model. The nanoparticles were conjugated with RGD (arginine-glycine-aspartic acid) short peptides that can specifically bind with the overexpressed $\alpha_{v} \beta_{3}$ integrin in the OSCC (36).

The superior anti-tumor effects of targeting the tumor site with the chemotherapeutic drugs through direct delivery encourage the ongoing studies in this field. Furthermore, loading the chemotherapeutics on nanoparticles is an emerging field in the drug delivery researches. The small size of the nanoparticles would reduce the amount of the loaded drug, with preserving its therapeutic effectiveness. In addition, the ease of nanoparticles to penetrate the capillaries would increase the concentration of the chemotherapeutic agent in the cancer cells (37).

\section{CONCLUSIONS}

The results of this study indicate that the intratumoral injection of OSCC with chemotherapeutic drug had a positive outcome and inhibited the growth of the tumors, with reduction in the tumor volume. The histological and the immunohistochemical studies proved the cytotoxic effect of DOX in the treatment of OSCC, by the increased $\mathrm{AI}$ and decreased proliferative activity.

\section{CONFLICT OF INTEREST}

The authors declare that they have no conflicts of interest.

\section{ACKNOWLEDGMENT}

The authors extend their appreciation to Associate Proffesor Heba Said Ramadan, Biophysics Department, Medical Research Institute, Alexandria University, Egypt, and Dr. Marwa Mounir Afifi, Lecturer of Oral Pathology, Faculty 
of Dentistry, Alexandria University, Egypt, for their great support and help to finish this study. This work was supported by the ALEX REP Foundation (Intelligent Therapeutic Nanomedicine: HLTH-13), Alexandria University Research Fund (AURF).

\section{REFERENCES}

1. Ferlay J, Shin H-R, Bray F, Forman D, Mathers C, Parkin DM. Estimates of worldwide burden of cancer in 2008: GLOBOCAN 2008. Int J cancer 2010;127:2893-917.

2. Popat K, McQueen K, Feeley TW. The global burden of cancer. Best Pract Res Clin Anaesthesiol. 2013;27:399408.

3. Pereira MC, Oliveira DT, Landman G, Kowalski LP. Histologic subtypes of oral squamous cell carcinoma: prognostic relevance. J Can Dent Assoc. 2007;73:339-44.

4. Gasco M, Crook T. The p53 network in head and neck cancer. Oral Oncol 2003;39:222-31.

5. Feller L, Lemmer J. Oral Squamous Cell Carcinoma: Epidemiology, Clinical Presentation and Treatment. J Cancer Ther. 2012;3:263-8.

6. Ma J, Liu Y, Huang X-L, Zhang Z-Y, Myers JN, Neskey $\mathrm{DM}$, et al. Induction chemotherapy decreases the rate of distant metastasis in patients with head and neck squamous cell carcinoma but does not improve survival or locoregional control: a meta-analysis. Oral Oncol. 2012;48:1076-84.

7. Logan RM. Advances in understanding of toxicities of treatment for head and neck cancer. Oral Oncol. 2009;45:844-8.

8. Shah JP, Gil Z. Current concepts in management of oral cancer - Surgery. Oral Oncol. 2009;45:394-401.

9. Thorn C, Oshiro C, Marsh S, Hernandez-Boussard T, McLeod $\mathrm{H}$, Klein $\mathrm{T}$, et al. Doxorubicin pathways: pharmacodynamics and adverse effects. Pharmacogenet Genomics. 2012;21:440-6.

10. Wang S, Konorev EA, Kotamraju S, Joseph J, Kalivendi $\mathrm{S}$, Kalyanaraman B. Doxorubicin induces apoptosis in normal and tumor cells via distinctly different mechanisms: Intermediacy of $\mathrm{H}_{2} \mathrm{O}_{2}$ - and p53-dependent pathways. J Biol Chem 2004;279:25535-43.

11. Parveen S, Misra R, Sahoo SK. Nanoparticles: A boon to drug delivery, therapeutics, diagnostics and imaging. Nanomedicine Nanotechnology, Biol Med. 2012;8:14766.

12. Rivera MCA. 4NQO carcinogenesis: A model of oral squamous cell carcinoma. Int J Morphol. 2012;30:30914.

13. Kitakawa D, Cabral LAG, Marques MEA, Salvadori DMF, Ribeiro DA. Medium-term tongue carcinogenesis assays: A comparative study between 4-nitroquinoline 1oxide (4NQO)-induced rat and dimethylbenzanthracene (DMBA)-induced hamster carcinogenesis. J Exp Anim Sci. 2006;43:219-27.

14. Keith WN, Mee PJ, Brown R. Response of Mouse Skin Tumors to Doxorubicin Is Dependent on Carcinogen Exposure. Cancer Res. 1990;50:6841-7.

15. Nelson JL, Roeder BL, Carmen JC, Roloff F, Pitt WG. Ultrasonically activated chemotherapeutic drug delivery in a rat model. Cancer Res. 2002;62:7280-3.

16. Afifi MM, El Sheikh SM, Abdelsalam MM, Ramadan H, Omar TA, El Tantawi M, et al. Therapeutic efficacy of plasmonic photothermal nanoparticles in hamster buccal pouch carcinoma. Oral Surg Oral Med Oral Pathol Oral
Radiol. 2013;115:743-51.

17. Cullen R, Maguire TM, McDermott EW, Hill ADK, O’Higgins NJ, Duffy MJ. Anti-apoptotic proteins, apoptotic and proliferative parameters and their prognostic significance in cervical carcinoma. Eur J Cancer. 2001;37:1104-10.

18. Okumura H, Natsugoe S, Nakashima S, Matsumoto M, Sakita H, Nakano S, et al. Apoptosis and cell proliferation in esophageal sqamous cell carcinoma treated by chemotherapy. Cancer Lett. 2000;158:211-6.

19. Myoung H, Kim MJ, Lee JH, Ok YJ, Paeng JY, Yun PY. Correlation of proliferative markers (Ki-67 and PCNA) with survival and lymph node metastasis in oral squamous cell carcinoma: a clinical and histopathological analysis of 113 patients. Int J Oral Maxillofac Surg. 2006;35:100510.

20. Ashkavandi ZJ, Najvani AD, Tadbir AA, Pardis S, Ranjbar MA, Ashraf MJ. MCM3 as a novel diagnostic marker in benign and malignant salivary gland tumors. Asian Pac J Cancer Prev. 2013;14:3479-82.

21. Oliveira LR, Ribeiro-Silva A. Prognostic significance of immunohistochemical biomarkers in oral squamous cell carcinoma. Int J Oral Maxillofac Surg. 2011;40:298-307.

22. Kim S. Animal models of cancer in the head and neck region. Clin Exp Otorhinolaryngol. 2009;2:55-60.

23. Surkin MI, Schwartz SA, Markiewicz DA. Late-onset complications after chemoradiation for head and neck carcinomas. Ear, Nose Throat J. 2013;92:18-24.

24. Toscano N, Holtzclaw D, Hargitai I. Oral Implications of Cancer Chemotherapy. J Implant Adv Clin Dent. 2009;1:51-69.

25. Matsumura Y, Maeda H. A New Concept for Macromolecular Therapeutics in Cancer Chemotherapy: Mechanism of Tumoritropic Accumulation of Proteins and the Antitumor Agent Smancs. Cancer Res. 1986;46:6387-92.

26. Maeda H, Wu J, Sawa T, Matsumura Y, Hori K. Tumor vascular permeability and the EPR effect in macromolecular therapeutics: A review. J Control Release. 2000;65:271-84.

27. Maeda H, Sawa T, Konno T. Mechanism of tumortargeted delivery of macromolecular drugs, including the EPR effect in solid tumor and clinical overview of the prototype polymeric drug SMANCS. J Control Release. 2001;74:47-61.

28. Maeda H. The enhanced permeability and retention (EPR) effect in tumor vasculature: The key role of tumorselective macromolecular drug targeting. Adv Enzyme Regul. 2001;41:189-207.

29. Yu MK, Park J, Jon S. Targeting strategies for multifunctional nanoparticles in cancer imaging and therapy. Theranostics. 2012;2:3-44.

30. Hu Z, Garen A. Intratumoral injection of adenoviral vectors encoding tumor-targeted immunoconjugates for cancer immunotherapy. PNAS. 2000;97:9221-5.

31. Slos P, Meyer M De, Leroy P, Rousseau C, Acres B. Immunotherapy of established tumors in mice by intratumoral injection of an adenovirus vector harboring the human IL-2 cDNA: Induction of CD8 + T-cell immunity and NK activity. Cancer Gene Ther. 2001;8:321-32.

32. Dubrot J, Palazón A, Alfaro C, Azpilikueta A, Ochoa MC, Rouzaut A, et al. Intratumoral injection of interferon- $\alpha$ and systemic delivery of agonist anti-CD137 monoclonal 
antibodies synergize for immunotherapy. Int $\mathrm{J}$ Cancer 2011;128:105-18.

33. Sakagami H. Apoptosis-inducing activity and tumorspecificity of antitumor agents against oral squamous cell carcinoma. JDSR. 2010;46:173-87.

34. Zhang L, Yu SU, Duan Z, Wang Q, Tian GE, Tian YAN, et al. Treatment of liver cancer in mice by the intratumoral injection of an octreotide-based temperature - sensitive gel. Int J Mol Med. 2014;33:117-27.

35. Shikanov A, Shikanov S, Vaisman B, Golenser J, Domb AJ. Cisplatin Tumor Biodistribution and Efficacy after Intratumoral Injection of a Biodegradable Extended Release Implant. Chemother Res Pract. 2011;2011:1-9.

36. Chen Y, Zhang W, Huang Y, Gao F, Fang X. In Vivo Biodistribution and Anti-Tumor Efficacy Evaluation of Doxorubicin and Paclitaxel-Loaded Pluronic Micelles Decorated with c ( RGDyK ) Peptide. PLoS One. 2016;11:1-18.

37. Kumar A, Zhang X, Liang X-J. Gold Nanoparticles: Emerging Paradigm for Targeted Drug Delivery System. Biotechnol Adv. 2013;31:593-606.

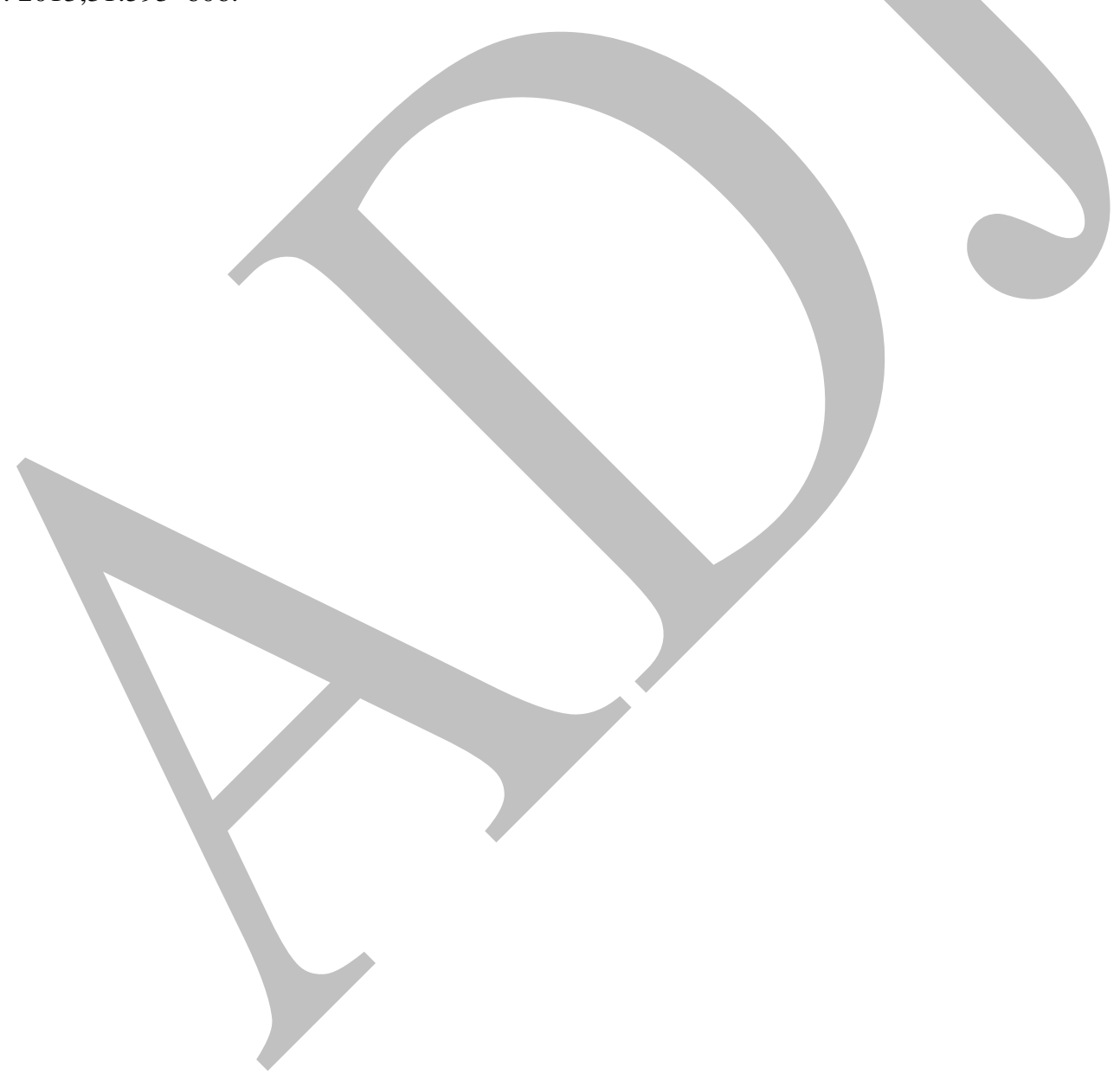

